\title{
PREVALENCIA DE LOS TRASTORNOS DEL ESPECTRO AUTISTA: REVISIÓN DE DATOS
}

\section{Prevalence of Autism Spectrum Disorders: data review}

\author{
Francisco Alcantud Marín \\ Universitat de Valencia. Centre Universitari de Diagnòstic i Atenció Primerenca. \\ Guardia Civil, 22 bajo. 46020 Valencia \\ francisco.alcantud@uv.es \\ Yurena Alonso EsTeban \\ Universitat de Valencia. Departamento de Psicología Evolutiva y de la Educación \\ Susana MATA ItURRAlde \\ Universidad de Guayaquil. Facultad de Ciencias Psicológicas. Instituto Nacional de Investigación \\ en Salud Pública Leopoldo Izquieta Pérez INSPI LIP
}

Recepción: 5 de octubre de 2016

Fecha de aceptación definitiva: 17 de enero de 2017

Resumen: Los datos publicados sobre la prevalencia de los Trastornos del Espectro Autista (TEA) apuntan hacia un incremento significativo de este indicador. Este incremento está siendo objeto de estudio en numerosas publicaciones de análisis, metaanálisis y revisiones sistemáticas. La prevalencia nos indica el porcentaje de personas que en un momento determinado padecen o son diagnosticadas de una enfermedad. Las consecuencias derivadas del incremento de la prevalencia son relevantes tanto desde el punto de vista sanitario, como social y educativo, pero sobre todo son relevantes cuando, como es el caso, no se conoce la causa del trastorno. Es en este sentido donde gana importancia el estudio de la prevalencia con la finalidad de acotar diversas circunstancias que puedan dar pistas sobre la posible causa o causas que generan los TEA. En este artículo se resumen los últimos estudios y revisiones y se reflexiona sobre los mismos y las posibles causas que justifiquen el incremento del que informan. Se analiza cómo estos indicadores epidemiológicos pueden o están influidos por posibles deficiencias metodológicas 
detrás de las cuales se puedan explicar las variaciones entre unos estudios y otros. Se concluye manifestando la necesidad de realizar en nuestro país estudios poblacionales y de seguimiento que nos permitan conocer la realidad de la evolución de estos trastornos con la finalidad de ofrecer información fehaciente a los responsables de las instituciones comprometidas con la detección y tratamiento de los TEA.

Palabras Clave: Trastornos del Espectro Autista; prevalencia; epidemiología.

Aвstract: Published data on the prevalence of autism spectrum disorders point to a significant increase in this indicator. This increase is being studied in numerous publications of analysis, meta-analysis and systematic reviews. The prevalence indicates the proportion of people who suffer at a given time or are diagnosed with a disease. The consequences of the increasing prevalence are relevant from the point of health, social and educational, but especially relevant when as is the case, the cause of the disorder is unknown. It is in this sense that the prevalence study gains importance in order to delimit various circumstances that may give clues to the possible cause or causes that generate disorder. This article reviews studies, summarizes the last data, and reflects on them and possible causes that justify the increased reporting. It looks like these epidemiological indicators can or are influenced by possible methodological flaws behind, which can explain the variations between studies and others. It concludes by stating the need population studies and monitoring that allows us to know the reality of the evolution of these disorders in order to provide reliable information to those responsible for the institutions involved in the detection and treatment of ASD.

KEYWORDs: Autism Spectrum Disorders; prevalence, epidemiology.

DeClARACiÓn DE INTERESES: Los autores manifiesta la inexistencia de conflictos de interés en relación con este artículo.

\section{Introducción}

L

os Trastornos del Espectro Autista (TEA) son trastornos del neurodesarrollo que presentan una gran variabilidad de síntomas (tanto en intensidad o gravedad como en diversidad). Se caracterizan, según el DSM-5 (APA, 2013), por tener en común déficit en la comunicación e interacción social y patrones de conducta restringidos por intereses y actividades repetitivas. Estos síntomas representan un continuo que, para alcanzar el diagnóstico, debe ser significativo y causar alteraciones y limitaciones en la vida diaria. Además, los síntomas deben estar presentes desde la primera infancia (antes de los 36 meses). Durante los últimos años se ha informado de dramáticos incrementos en la prevalencia de los TEA. Muchas publicaciones corroboran la tendencia al alza de este indicador desde 4-5/10.000 en los años sesenta hasta 260/10.000 o más en las primeras décadas del siglo XXI (Matson y Kozlowski, 2011).

(C) Ediciones Universidad de Salamanca / CC BY-NC-ND

Siglo Cero, vol. 47 (4), n. ${ }^{\circ} 260,2016$, octubre-diciembre, pp. 7-26 
La prevalencia de los TEA ha sido un tema controvertido por las implicaciones que conlleva. En particular, el hecho de que aún hoy no son conocidas las causas que lo originan hace que la obtención de valores epidemiológicos y la comparación adecuada entre zonas geográficas, con otras patologías, edades, etc., sea un primer paso descriptivo para lanzar hipótesis de trabajo sobre factores potencialmente causales que pudieran ser contrastados de forma experimental o clínica.

La razón de las variaciones en los resultados de la prevalencia de los TEA es motivo de debate constante. Existen quienes apuntan a que los TEA son trastornos con una incidencia creciente (Blaxill, 2004); otros muchos apuntan que, reconociendo el incremento, lo explican parcialmente por diversas razones de orden metodológico, como los cambios de criterios diagnósticos o la mayor divulgación y conocimiento de la sintomatología entre profesionales y familiares o el desarrollo de mejores métodos de detección (Fombonne, 2003; Charman, 2002; Wing y Potter, 2002; Coo y otros, 2008). No obstante, con problemas metodológicos o sin ellos, todos evidencian un crecimiento de la prevalencia más o menos significativo en los últimos 50-60 años. De ser ciertos los datos de prevalencia que actualmente se manejan (Baio, 2012; Baio, 2014; Chistensen y otros, 2016), significaría que hay más niños con TEA que afectados por cáncer, diabetes juvenil, fibrosis quística y distrofia muscular juntos (Klin, Klaiman y Jones, 2015).

\section{Objetivo}

En este artículo intentaremos analizar los últimos estudios de prevalencia y las posibles y diversas razones que pueden estar detrás del incremento de este indicador epidemiológico, tanto desde el punto de vista metodológico como desde otros puntos de vista.

\section{Desarrollo}

\subsection{Los datos}

La primera revisión crítica de publicaciones sobre la prevalencia de los TEA que conocemos es debida a Wing (Wing, 1993), en la que revisa un total de dieciséis estudios de prevalencia en los que se pueden comparar los resultados por utilizar métodos de búsqueda y estimación similares o equivalentes, el más antiguo de ellos es el de Lotter de 1966 (Lotter, 1966) y el más moderno el estudio de Gillberg y colaboradores de 1991 (Gillberg, Steffenburg y Schaumann, 1991). En esta revisión se incluyen estudios de Inglaterra (2), Japón (5), USA (2), Suecia (4) y Dinamarca, Canadá y Francia (1). Las tasas específicas variaron desde 3,3 hasta 16,0 por $10.000(6,5 / 10.000$ de promedio). Para Wing (1993) todas las diferencias podrían deberse a variaciones en la práctica de diagnóstico y al incremento en el conocimiento de las manifestaciones del autismo. 
Por otra parte, la revisión sistemática más reciente a la que hemos tenido acceso es la realizada por Hill, Zuckerman y Fombonne que, desde el año 2000 hasta el 2014, encuentran un total de 53 estudios en 18 países diferentes [UK (14), USA (13), Suecia (5), Canadá (4), Australia (2), Dinamarca (2), Noruega (2), China (1), Venezuela (1) Japón (1), Holanda (1), Omán (1), Corea del Sur (1), Finlandia (1), Irán (1), Taiwán (1), Israel (1) e Islandia (1)], con tamaños muestrales entre 5.000 y 4,5 millones de personas. La mayoría de los estudios recogidos en esta revisión fueron publicados en el 2009 o después (Hill, Zuckerman y Fombonne, 2015). Aunque la mayoría de los estudios de esta revisión podríamos decir que son recientes, resulta curiosa la variabilidad de criterios diagnósticos utilizados (CIE, 8,9 y 10, DSM IV, DSM TV-R y otros). Por restricciones en la búsqueda todos los trabajos debían haber sido publicados en inglés; este hecho puede justificar que la inmensa mayoría de estudios sean anglosajones. Entre los datos de prevalencia, varían tanto en su magnitud, desde 6,4/10.000 en Irán (Samadi, Mahmoodizadeh y McConkey, 2012) hasta el mayor índice de prevalencia 264/10.000 en Corea del Sur (Kim y otros, 2011), como en la metodología utilizada. Para los autores, los resultados respaldan la idea de que la incidencia actual de TEA ronda el $0,69 \%$ o $1 / 145$ niños. Aunque evidencian un incremento en la tasa de prevalencia no se puede atribuir exclusivamente a un incremento de la incidencia. En las conclusiones, estos autores apuntan hacia la idea de que la tendencia al alza del índice de prevalencia del trastorno responde a los cambios de criterios diagnósticos, a la evolución de las prácticas de detección y diagnóstico, al incremento en la disponibilidad de servicios de atención y en general a un mayor conocimiento del trastorno. Así, por ejemplo, la expansión del aumento de la prevalencia observada en muchos países durante la década de 1990-2000 se corresponde también con una expansión de servicios en esos países. Sin embargo, sin duda los servicios son consecuencia de la presión social que los demanda y no al contrario. En un estudio reciente (Mandell y otros, 2016) se analiza la influencia de la legislación desarrollada en algún estado de USA que obliga a incluir en las pólizas de seguros sanitarios los tratamientos de los niños con TEA. Según sus datos de seguimiento, en los estados donde se puso en marcha esta norma legal se produjo un incremento medio de prevalencia del 0,17/1000 en el primer año, $0,27 / 1000$ en el segundo y 0,29/1000 en el tercero. Concluyen que la incorporación de los servicios de intervención a niños con TEA en las pólizas de seguros incrementa el número de niños que se diagnostican. No obstante, se puede asumir que tanto las compañías de seguros como los políticos que legislan sobre la conveniencia o no de incluir en las pólizas de seguros unos u otros tratamientos no lo harían si no existiera una demanda social objetiva.

Los resultados de un estudio más reciente realizado en Suecia (Lundström, Reichenberg, Anckarsäter, Lichtemstein y Gillberg, 2015), analizaron más de un millón de niños (aproximadamente 4.620 con TEA) nacidos entre 1993 y 2002, respaldan la idea de que el incremento en la tasa de prevalencia responde a un mayor conocimiento del trastorno. En este estudio, el número de diagnósticos aumentó de 1/435 a 1/200. La incidencia de los TEA, sin embargo, se mantuvo contante en torno a 1/105. En estudios similares al anterior pero en Dinamarca (Hansen, Schendel y Parner, 2014) y también en Reino Unido (Taylor, Jick y MacLaughlin, 2013) mostraban como la

(C) Ediciones Universidad de Salamanca / CC BY-NC-ND

Siglo Cero, vol. 47 (4), n. ${ }^{\circ}$ 260, 2016, octubre-diciembre, pp. 7-26 
incidencia se mantuvo más o menos estable de 2004 a 2010 oscilando en función de los estudios entre $1 / 150$ y $1 / 175$.

TABla 1. Resultados de prevalencia de los Trastornos del Espectro Autista (Baio, 2012; Baio, 2014) http://www.cdc.gov/ncbddd/autism/data.html (consultada 21-3-2016)

\begin{tabular}{|c|c|c|c|c|}
\hline & $\begin{array}{c}\text { Población total } \\
\text { de niños/as } \\
\text { de vinos bajo } \\
\text { vigilancia }\end{array}$ & $\begin{array}{c}\text { Prevalencia TEA } \\
\text { por 1,000 } \\
\text { (intervalo de } \\
\text { de confianza 95\%) }\end{array}$ & $\begin{array}{c}\text { Aproximadamente } \\
1 / x \text { niños }\end{array}$ \\
\hline 2000 & 1992 & 187.761 & $6,7(6,3-7,1)$ & $1 / 150$ \\
\hline 2002 & 1994 & 407.578 & $6,6(6,4-6,9)$ & $1 / 150$ \\
\hline 2004 & 1996 & 172.335 & $8,0(7,6-8,4)$ & $1 / 125$ \\
\hline 2006 & 1998 & 307.790 & $9,0(8,6-9,3)$ & $1 / 110$ \\
\hline 2008 & 2000 & 337.093 & $11,3(11,0-11,7)$ & $1 / 88$ \\
\hline 2010 & 2002 & 363.749 & $14,7(14,3-15,1)$ & $1 / 68$ \\
\hline 2012 & 2004 & 346.978 & $14,6(8,2-24,6)$ & $1 / 68$ \\
\hline
\end{tabular}

Por poner el acento en el problema, nos centraremos en los últimos años y en una única fuente, así el $\mathrm{CDC}^{1}$ dispone de una red de vigilancia y supervisión de los TEA y trastornos del desarrollo en general $(\mathrm{ADDM})^{2}$. Esta red publica periódicamente estudios comparables entre sí por las características metodológicas utilizadas (Baio, 2012; Baio, 2014; Chistensen y otros, 2016). En la Tabla 1, se muestra que la prevalencia de niños afectados por TEA ha aumentado en los últimos años respecto a los primeros de la serie y, más aún, si los comparamos con la revisión realizada por Wing (Wing, The definition and Prevalence of Autism: A Review, 1993). Para evitar las críticas metodológicas, en estos estudios de seguimiento se han tomado diferentes años de control (cada dos años desde el año 2000) y se contabiliza cuántos niños diagnosticados existen que cumplan los ocho años de edad (cohorte de edad). Se optó por tomar esta edad, dos años después del inicio de la escolarización obligatoria, por considerar que el diagnóstico debía ya ser estable. De los datos de la Tabla 1 se desprende que existe un incremento significativo en el número de niños con TEA. El incremento es indudable desde índices de prevalencia obtenidos por Lotter de 4,5/10.000 niños (Lotter, 1966; Lotter, 1967), 15/10.000 obtenidos por Wing y Gould (Wing y Gould, 1979) hasta los encontrados por Baio (Baio, 2012; Baio, 2014; Chistensen y otros, 2016). Es de destacar que los datos de la última edición del CDC convergen con los obtenidos en el estudio poblacional de Corea del Sur (Kim y otros, 2011).

Una constante en todos los estudios es la mayor prevalencia de los TEA en niños varones. La ratio oscila entre un caso de niña cada 4-5 niños. También los estudios demuestran que no existen diferencias sociales ni étnicas, aunque en los estudios del CDC se da a entender que en USA existe más probabilidad de detectar TEA en población

CDC's: Centers for Disease Control and Prevention's.

2 ADDM: Autism and Developmental Disabilities Monitoring Network.

(C) Ediciones Universidad de Salamanca / CC BY-NC-ND

Siglo Cero, vol. 47 (4), n. ${ }^{\circ}$ 260, 2016, octubre-diciembre, pp. 7-26 
hispana o afroamericana. En todo caso, estas diferencias deben atribuirse a los sistemas de diagnóstico o de muestreo en los puntos de recogida de la información (Boat y Wu, 2015). Debemos hacer constar que los TEA se identifican por un déficit en comunicación e interacción social, por lo que grupos socialmente excluidos o con normas sociales no convencionales podrían ser más sensibles a los criterios diagnósticos generando posibles falsos positivos. Por poner un ejemplo, al comparar la prevalencia entre población aborigen en Australia, se detectan prevalencias diferentes en Discapacidad Intelectual y TEA (3,9\% en población aborigen frente a $1,57 \%$ en población no aborigen) (Bourke, de Klerk, Smith y Leonard, 2016).

En nuestro país disponemos de escasa o nula información de estudios poblacionales (Posada-De la Paz, Ferrari-Arroyo, Touriño y Boada, 2005). Los pocos estudios que conocemos son resultado de iniciativas locales sobre muestras limitadas o estudios ex-post-facto sobre las historias clínicas ya realizadas. El estudio más antiguo que conocemos es el realizado en la Comunidad de Navarra (Alberdi, 1990), que realiza una consulta a las historias clínicas y determina una prevalencia de 4,8/10.000. El último y quizás el más amplio y con mayores fortalezas metodológicas es el realizado en la Comunidad Autónoma de Canarias (Fortea, Escandell y Castro, 2013). En este estudio sobre una muestra de 1.796 niños de 18 a 36 meses de Las Palmas los padres contestaron el cuestionario M-CHAT/ES y, posteriormente, se confirmó el diagnóstico mediante la entrevista ADI-R (Rutter, Le Couteur y Lord, 2009) y la escala de observación ADOS2 (Lord y otros, 2012). La prevalencia estimada es del 0,61\% (1/164) en línea con los resultados de otros estudios internacionales. El dato diferencial de este estudio es la mayor prevalencia del trastorno en niñas (6 niñas por cada 5 niños) debido posiblemente al tamaño muestral. En el estudio de validación de la versión española del M-CHAT (Canal y otros, 2010) obtuvieron tasas similares (1/156, es decir, 0,64\%).

TABLA 2. Relación de estudios de prevalencia realizados en España (elaboración propia)

\begin{tabular}{|l|l|c|l|l|c|}
\hline \multicolumn{1}{|c|}{ Autores } & \multicolumn{1}{|c|}{ Lugar } & Edad & \multicolumn{1}{|c|}{ Método } & $\begin{array}{c}\text { Criterios } \\
\text { diagnósticos }\end{array}$ & $\begin{array}{c}\text { Prevalencia } \\
110000\end{array}$ \\
\hline Alberdi, 1990 & Navarra & $0-15$ & $\begin{array}{l}\text { Revisión historias } \\
\text { clínicas }\end{array}$ & DSM-III & 4,8 \\
\hline Garanto, 1994 & Cataluña & $4-17$ & $\begin{array}{l}\text { Revisión historias } \\
\text { clínicas }\end{array}$ & 2,49 \\
\hline Orte y otros, 1995 & Murcia & $0-16$ & & DSM-III & 2,93 \\
\hline Folch y Sanchez, 1998 & Cataluña & $0-99$ & $\begin{array}{l}\text { Revisión historias } \\
\text { clínicas }\end{array}$ & $\begin{array}{l}\text { DSM-IV y } \\
\text { CIE-10 }\end{array}$ & 6,1 \\
\hline $\begin{array}{l}\text { Fuente especificada } \\
\text { no válida }\end{array}$ & Madrid & & & DSM-IV & 8,34 \\
\hline Frontera, 2005 & Aragón & $0-16$ & $\begin{array}{l}\text { Revisión historias } \\
\text { clínicas }\end{array}$ & $\begin{array}{l}\text { Diagnóstico } \\
\text { Previo }\end{array}$ & 9,21 \\
\hline $\begin{array}{l}\text { Sanchez-Valle } \\
\text { y otros, 2008 }\end{array}$ & Nacional & $0-14$ & $\begin{array}{l}\text { Estimación según } \\
\text { modelo teórico }\end{array}$ & & 2,51 \\
\hline
\end{tabular}

() Ediciones Universidad de Salamanca / CC BY-NC-ND

Siglo Cero, vol. 47 (4), n. ${ }^{\circ}$ 260, 2016, octubre-diciembre, pp. 7-26 
PREVALENCIA DE LOS TRASTORNOS DEL ESPECTRO AUTISTA: REVISIÓN DE DATOS FRANCISCO ALCANTUD MARÍN, YURENA ALONSO ESTEBAN Y SUSANA MATA ITURRALDE

\begin{tabular}{|l|l|c|l|l|c|}
\hline \multicolumn{1}{|c|}{ Tublares 2 Relación de estudios de prevalencia realizados en España (elaboración propia) } \\
\hline $\begin{array}{l}\text { Trujillo, Sánchez } \\
\text { y Gutiérrez, 2007 }\end{array}$ & Cádiz & $2-14$ & $\begin{array}{l}\text { Revisión historias } \\
\text { clínicas }\end{array}$ & $\begin{array}{l}\text { CIE-10 y } \\
\text { diagnósticos }\end{array}$ & $\begin{array}{c}\text { Prevalencia } \\
110.000\end{array}$ \\
\hline $\begin{array}{l}\text { Canal y otros, 2010 } \\
\text { García-Primo y otros, } \\
2014\end{array}$ & $\begin{array}{l}\text { Salamanca } \\
\text { y Zamora }\end{array}$ & $\begin{array}{l}18-36 \\
\text { meses }\end{array}$ & $\begin{array}{l}\text { Detección } \\
\text { y diagnóstico } \\
\text { ADOS-G }\end{array}$ & DSM IV-TR & $642 \%$ \\
\hline $\begin{array}{l}\text { Fortea, Escandell } \\
\text { y Castro, 2013 }\end{array}$ & Canarias & $\begin{array}{l}18-36 \\
\text { meses }\end{array}$ & $\begin{array}{l}\text { Detección } \\
\text { y diagnóstico } \\
\text { ADI-R + ADOS }\end{array}$ & DSM-IV-TR & 61 \\
\hline
\end{tabular}

Otros datos son los ofrecidos por instituciones oficiales, así según el IMSERSO 3 (IMSERSO, 2005) en España existían en 2005 un total de 25.000 personas diagnosticadas con TEA. Según la Encuesta de Discapacidad, Autonomía Personal y Situaciones de Dependencia realizada por el INE4 en el año 2008 (INE, 2011; INE 2011), existen 13.800 personas de 6 años o más y 1.900 de menos de 5 años con un diagnóstico de autismo. Estos datos arrojan una prevalencia muy por debajo de las obtenidas por los estudios que aparecen en la Tabla 2.

FIGURA 1. Datos de prevalencia en función de la etapa educativa. Gráfica basada en datos tomados de Saldaña y otros, 2013

\begin{tabular}{|c|c|c|c|c|c|}
\hline \multirow{3}{*}{$\begin{array}{l}25 \\
20\end{array}$} & \multicolumn{5}{|c|}{ Prevalencia (por 10.000) } \\
\hline & 23,05 & & & & \\
\hline & & 16,5 & & & \\
\hline 10 & & & 9,7 & & \\
\hline & & & & 4,5 & \\
\hline$J$ & & & & & 0,75 \\
\hline & Infantil & Primaria & $\begin{array}{l}\text { Secundaria } \\
\text { obligatoria }\end{array}$ & $\begin{array}{c}\text { Secundaria } \\
\text { postobligatoria }\end{array}$ & Etapa adulta \\
\hline
\end{tabular}

3 IMSERSO: Instituto de Mayores y Servicios Sociales dependiente del Ministerio de Sanidad, Servicios Sociales e Igualdad.

4 INE: Instituto Nacional de Estadística. Ministerio de Economía y Competitividad.

(C) Ediciones Universidad de Salamanca / CC BY-NC-ND

Siglo Cero, vol. 47 (4), n. ${ }^{\circ} 260,2016$, octubre-diciembre, pp. 7-26

$$
-13-
$$


En un estudio sobre la vida adulta y TEA desarrollado por Autismo Andalucía (Saldaña y otros, 2013), se obtienen resultados de prevalencia que se aproximan a las cifras internacionales solo en la detección temprana actual. Tal como se observa en la Figura 1 el número de casos encontrados en el estudio citado es mucho mayor en la etapa infantil que en la edad adulta. Esta tendencia también se observa en el estudio realizado en Cádiz (Trujillo, Sánchez y Gutiérrez, 2007). La lectura positiva es que se han mejorado significativamente los procesos de detección. También existe una lectura negativa, a saber, la baja tasa de prevalencia en las etapas educativas intermedias y sobre todo en la edad adulta no parece reflejar la prevalencia real de la población con TEA. Es posible que una gran cantidad de casos se encuentren erróneamente diagnosticados como discapacidad intelectual (retaso mental) o incluso, peor aún, como algún tipo de trastorno mental (esquizofrenia, psicosis, etc.). Muchas personas con TEA adultas pueden haber sido olvidadas en instituciones psiquiátricas con tratamiento de psicofármacos de trastornos que nunca tuvieron (Lai y Baron-Cohen, 2015).

\subsection{Origen de las diferencias entre estudios}

De los datos aportados en el epígrafe anterior y en particular de la revisión por Hill, Zuckerman y Fombonne (Hill, Zuckerman y Fombonne, 2015) se desprende que el tipo de estudio influye directamente en el valor de la prevalencia. Así, estudios de revisión de casos realizados sobre expedientes (ex-post-facto) y en países con bajo desarrollo económico o poca trasparencia en la información clínica tienden a minusvalorar la prevalencia. Este es el caso, por ejemplo, de los estudios desarrollados en Irán (Sadami, Mahmoodizadeh y McConkey, 2012; Sadami y McConkey, 2011), Venezuela (Montiel-Nava y Peña, 2008) o en China (Wong y Hui, 2008), que informan de valores inferiores a 20/10.000. La relación entre calidad de la recogida de información y resultados se evidencia en el estudio de Omán, que obtiene solo un 1,4/10000 (Al-Farsi, Al Farsi y Brooks, 2011). Es posible, como se ha dicho con anterioridad, que los síntomas diagnósticos puedan estar influidos por normas sociales y culturales (Ouhtit y otros, 2015). Frente a estos estudios de revisión de casos en expedientes o registros oficiales con o sin contraste clínico, se encuentran con máxima prevalencia los estudios clínicos poblacionales, quizás el más importante de ellos es el estudio realizado en Corea del Sur (Kim y otros, 2011) en el que se detectan un total de 264/10000 casos.

Al margen de que el desarrollo de los países influya en los recursos dedicados tanto a la detección y diagnóstico como a la calidad de los sistemas de información, se debe contrastar la hipótesis de que según localizaciones geográficas pueda existir una tasa mayor de prevalencia de TEA. El interés de estos estudios diferenciales es hacer covariar la prevalencia con factores ambientales (contaminación, clima, usos culturales, etc.) que den pistas sobre la etiología de los TEA. Si tomamos como referencia los datos del CDC y su descomposición según los diferentes estados de USA, se observa una gran variación entre unos y otros, presentándose así resultados tan dispares como 1/175 en Alabama frente a 1/45 en Nueva Jersey (Baio, 2014). Sin 
embargo, estudios posteriores atribuyen las diferencias entre estados a las diferentes fuentes de información utilizadas, siendo significativamente menor la prevalencia de TEA estimada en los estados en el que se tuvo acceso solo a fuentes de datos de salud en comparación con la de los estados en los que también se disponía de datos educativos (Boat Wu, 2015).

Al analizar y comparar estudios muestrales se pueden apreciar diferencias entre unos y otros, incluso entre aquellos en los que se utilizan la misma metodología de muestreo y criterios diagnósticos. Estas diferencias pueden deberse a que de una única población pueden salir infinitas muestras, por tanto, la mejor estimación del estadístico poblacional sería el promedio entre todas las muestras obtenidas. Chakrabarti y Fombonne realizaron dos estudios separados entre 1992 y 1995 en Staffordshire (UK) con una metodología rigurosamente idéntica. Los resultados de la comparación de estos estudios sugieren que las diferencias al alza obtenidas no son estadísticamente significativas, lo que contradice el estudio de Göteborg en el que en tres ediciones consecutivas del mismo estudio se obtienen incrementos de la prevalencia 1989 (4/10.000); 1984 (6,6/10.000) y 1988 (9,5/10.000) (Gillberg, 1984; Gillberg, Steffenburg y Schaumann, 1991). Para determinar tendencias se debería disponer de sistemas de recogida de información mucho más exactos y observaciones a más largo plazo de tiempo.

Además del tipo de estudio y muestreo en la determinación de este índice influyen tres elementos fundamentales: criterios diagnósticos, la identificación de los casos y los métodos de evaluación (Fombonne, 2007).

\subsection{Criterios diagnósticos}

Aunque hoy en día existe fiabilidad en el diagnóstico de los TEA, aún persisten diferencias en la nomenclatura, la terminología y la operacionalización de los criterios en función del uso de las diferentes normas internacionales de diagnóstico (DSM-5, CIE-10, etc.). Es evidente que los cambios de criterio influyen en la definición de casos en los estudios epidemiológicos y, consecuentemente, en la estimación de la prevalencia de los mismos. La combinación de la determinación del caso con el tipo de metodología puede inducir a error como, por ejemplo, en los estudios documentales retrospectivos en los que se utilizaron diferentes criterios diagnósticos.

La gráfica de la Figura 2 muestra los resultados de diferentes estudios realizados aplicando criterios diagnósticos o de determinación de casos diferentes a lo largo de los últimos setenta años (Rice, 2013). En la figura, se muestra la coincidencia de incrementos en la prevalencia con los cambios introducidos a final de siglo XX con la publicación del CIE-10 y el DSM-IV. En un estudio reciente (Christiansz, Gray, Taffe y Tonge, 2016) se comparan 185 niños diagnosticados como TGD según el DSM-IV de edades comprendidas entre 20 y 55 meses, con los criterios diagnósticos actuales de TEA del DSM-5. Como instrumentos de diagnóstico se emplearon la información combinada del ADI-R y ADOS. Los resultados obtenidos manifiestan una sensibilidad de 0,84 y una especificidad de 0,54. Se constata, en consecuencia, que en el futuro existirán variaciones de diagnóstico al aplicar los nuevos criterios del DSM-5. 


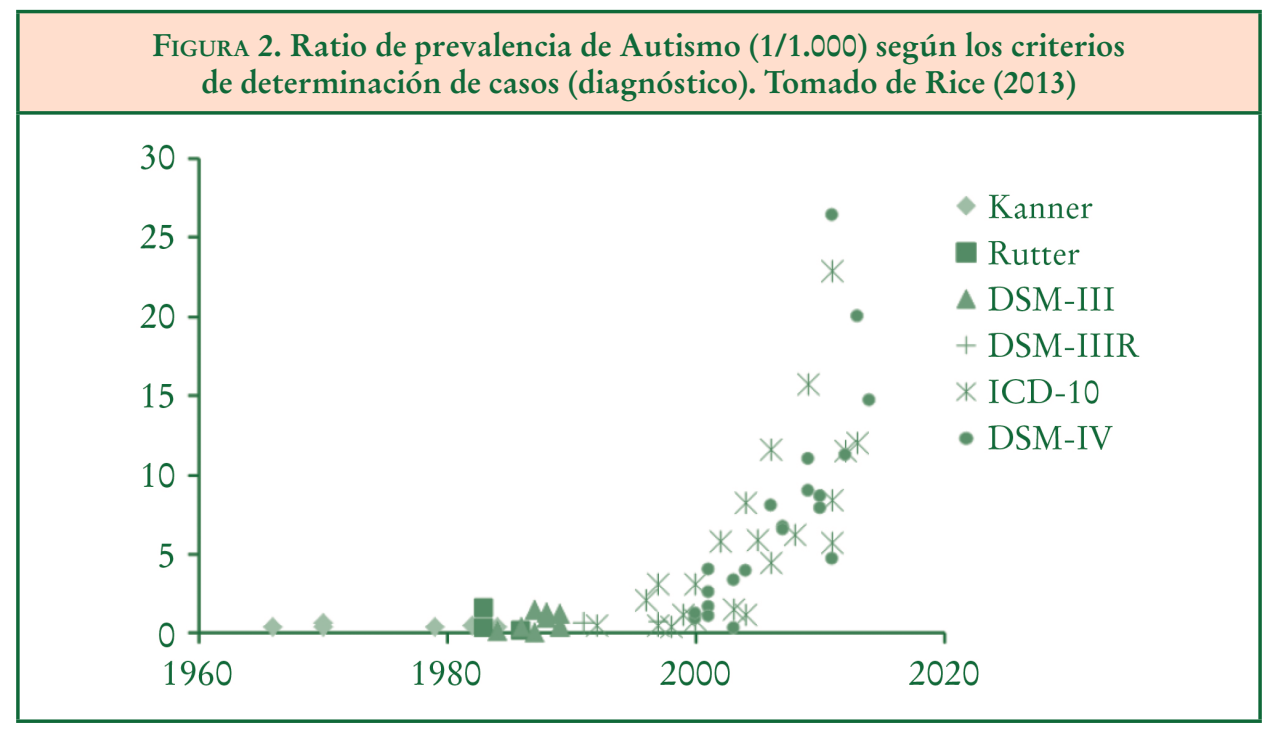

\subsection{Métodos de identificación de los casos}

Cuando una población tiene un tamaño o una dispersión que resulta inabordable se recurre a la determinación de muestras que puede basarse en el uso de registros de entidades que proveen servicios a la población objeto de estudio (p. e. bases de datos de servicios sanitarios, servicios de educación especial, servicios sociales, registros locales o nacionales, etc.). Este tipo de estudios tiene como limitación la suposición de que todas las personas afectadas por el trastorno acceden a los servicios y, por tanto, es posible que se subestime la prevalencia, sobre todo en comunidades donde son reconocidas las limitaciones en los servicios disponibles (por ejemplo, zonas rurales o de difícil acceso), o en aquellas comunidades donde los servicios se han implantado recientemente y por tanto aún no tienen historia.

En Estados Unidos, varios estudios han podido disponer de información proveniente de diferentes fuentes de este tipo. En base a los datos educativos del estado de California (Cavagnaro, 2007) se realizaron estudios de prevalencia entre 1987 y 2007, observándose un incremento muy significativo. En un estudio posterior presentado por Boat y Wu (Boat y Wu, 2015), los índices oscilaron entre el 1,7/1000 en el 2001 y el 7,6/1000 en el 2012 (ver imagen de la Figura 3).

$\mathrm{Al}$ margen de los datos oficiales ofrecidos por los gobiernos también se dispone de información obtenida por encuesta. Según los datos de la encuesta NHIS (Boyle y otros, 2011), la prevalencia ha crecido en USA de 1,9/1000 en 1997-1999 hasta un 7,4 /1000 en el periodo 2006-2008 (ver Figura 4). En la Encuesta Nacional de Salud Pediátrica $(\mathrm{NSCH})^{5}$ de USA, se incluyeron ítems relacionados con los TEA, lo que

5 National Survey of Children's Health.

(C) Ediciones Universidad de Salamanca / CC BY-NC-ND

Siglo Cero, vol. 47 (4), n. ${ }^{\circ} 260,2016$, octubre-diciembre, pp. 7-26 
permitió observar un incremento en la prevalencia del diagnóstico de TEA entre los años 2003 y 2012, de un 5,5/1000 hasta un 11,6/1000 (Blumberg y otros, 2013).

FiguRA 3. Número de niños americanos que reciben atención por Autismo en los servicios de Educación Especial (tanto por mil). Gráfica desarrollada en base a los datos ofrecidos por Boat y Wu (2015)

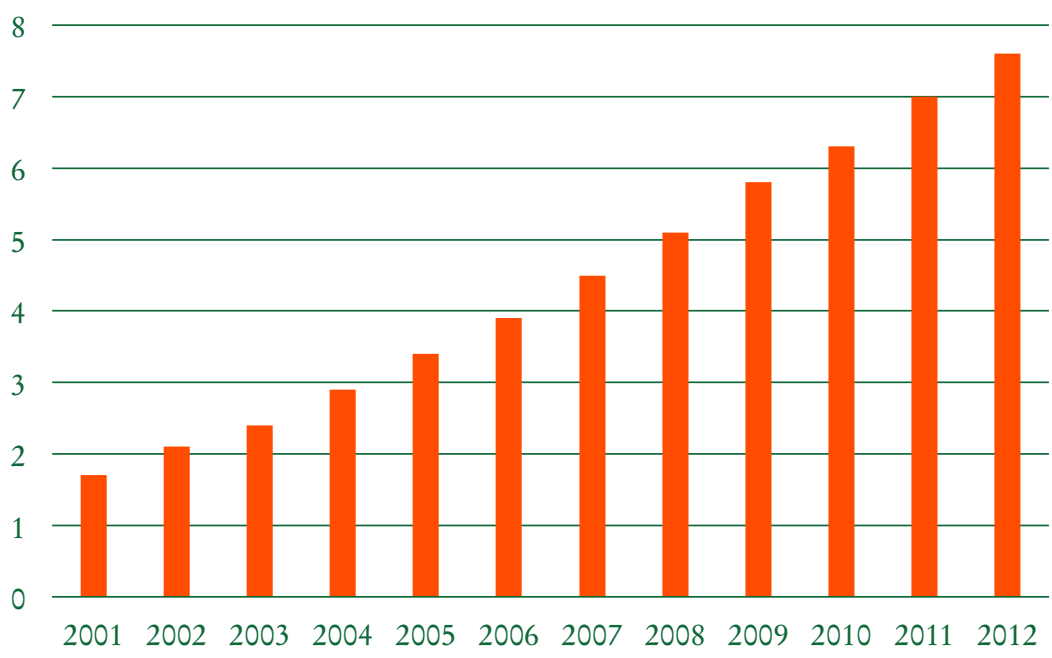

Figura 4. Tendencia de la prevalencia en USA según la Encuesta Nacional de Salud. Gráfica desarrollada en base a los datos ofrecidos por Boyle y otros (2011)

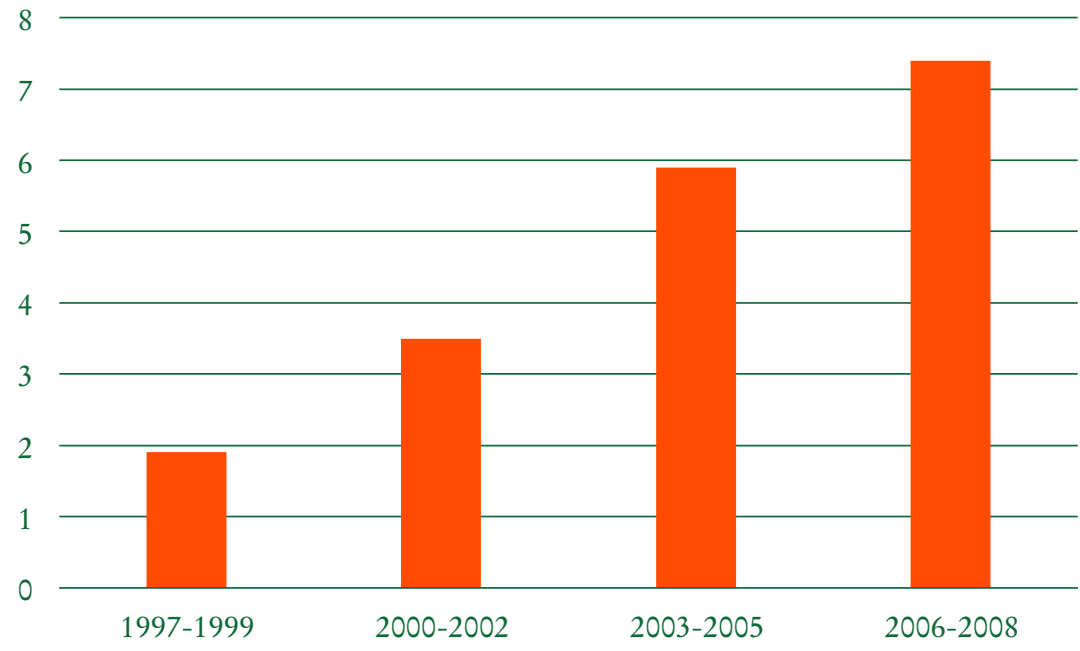

(C) Ediciones Universidad de Salamanca / CC BY-NC-ND

Siglo Cero, vol. 47 (4), n. ${ }^{\circ}$ 260, 2016, octubre-diciembre, pp. 7-26

$$
-17-
$$




\subsection{Análisis de los cambios de tendencia}

En los datos de los estudios mostrados con anterioridad (Cavagnaro, 2007; Boyle y otros, 2011; Boat y Wu, 2015) se desprende que existe en la serie histórica una tendencia secular positiva en las tasas de prevalencia del autismo. Este hecho se ha interpretado como un incremento de la incidencia del trastorno.

Como se señaló anteriormente, la prevalencia y la incidencia aumentan cuando la definición de casos se amplía o los procedimientos de diagnóstico, detección o determinación de los casos se mejoran. Las tendencias temporales de las estimaciones de prevalencia sólo pueden ser evaluadas en las investigaciones que tienen parámetros metodológicos estrictos y homogéneos. Así en los estudios del CDC (Baio, 2012; Baio, 2014; Chistensen y otros, 2016) se han unificado criterios de edad, muestreo y forma de diagnóstico. No obstante, se debe caer en la cuenta de que son estudios muestrales.

Uno de los datos utilizados para estimar la prevalencia es el número de niños que reciben atención en los servicios especializados sanitarios o de educación especial. Las tendencias al alza de estos datos se han detectado en muchos países (Shattuck, 2006; Lotter, Epidemiology of autistic conditions in young children: I Prevalence, 1966; Taylor, Jick y MacLaughlin, 2013). Sin embargo, las tendencias al alza de estos datos pueden estar influenciadas por la mayor disponibilidad de servicios; mayor concienciación pública y consecuentemente mayor dotación económica; la disminución de la edad en el momento del diagnóstico, y los cambios con el tiempo en los conceptos y prácticas del mismo.

Otra posible explicación para el aumento de la prevalencia en una categoría diagnóstica es la sustitución de otra categoría diagnóstica o la comorbilidad de síntomas. Esta sustitución de diagnóstico (o conmutación) puede ocurrir cuando las categorías de diagnóstico se hacen más familiares para los profesionales y/o cuando esta nueva categoría diagnóstica puede facilitar o garantizar el acceso a mejores servicios.

La evidencia más fuerte de cómo la sustitución de diagnóstico contribuye a aumentar la prevalencia de TEA se demostró con el análisis de los datos del Departamento de Educación de USA (Shattuck, 2006). En este estudio, se demuestra que mientras la prevalencia de la discapacidad intelectual (RM) disminuye año a año, la de los TEA se incrementa. Sobre todo, el cambio de tendencia se observa desde que el diagnóstico TEA se convirtió en una categoría diagnóstica independiente.

Otra evidencia en esta misma dirección son los resultados del estudio epidemiológico sobre niños de 8 años en el área metropolitana de Atlanta durante el periodo 1996 a 2010 (Braun y otros, 2015), donde las tendencias de las prevalencias de DI (Discapacidad Intelectual) y TEA se invierten con el paso del tiempo (ver Figura 5).

En la misma dirección los resultados del análisis de las solicitudes de subsidio del sistema SSA ${ }^{6}$ por trastorno mental apuntan una clara tendencia en los últimos años. En la última década el TDAH es y era el trastorno mayoritario por el que se accedía a una ayuda económica. Se ha observado como los TEA han ido incrementado el número de solicitudes mientras que el resto de categorías ha decrecido o se ha mantenido

6 SSA: Social Security Administration. http://www.ssa.gov.

(C) Ediciones Universidad de Salamanca / CC BY-NC-ND

Siglo Cero, vol. 47 (4), n. ${ }^{\circ}$ 260, 2016, octubre-diciembre, pp. 7-26 
FIGURA 5. Contraste de tendencias de la prevalencia entre DI y TEA (Fuente 58)

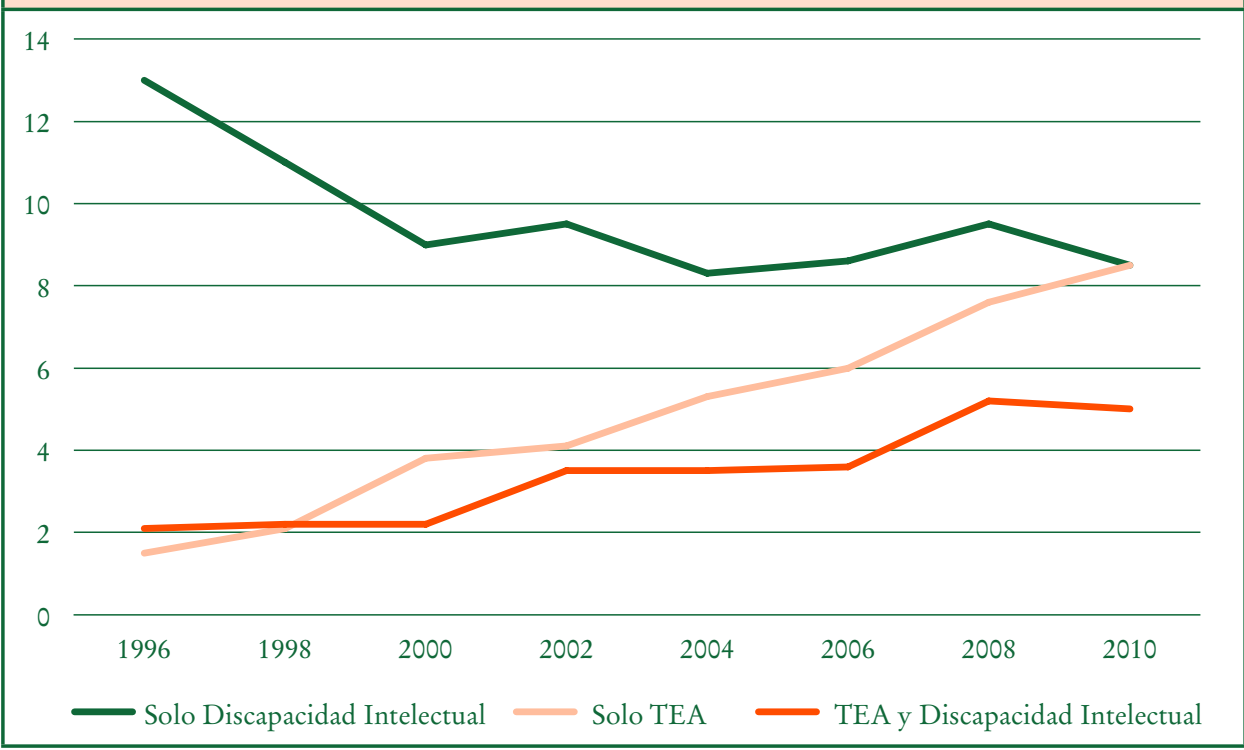

constante. Debe tenerse en cuenta la no correspondencia estricta entre las categorías diagnósticas del DSM-5 y las categorías que origina la percepción de una pensión (Boat y Wu, 2015) (ver Figura 6).

Utilizando datos individuales, King y Bearmen analizan la hipótesis de sustitución llegando a la conclusión de que, en el estado de California, el 24\% del aumento de casos de TEA fue debido a una permuta en el diagnóstico o a la acumulación de diagnóstico por comorbilidad (King y Bearman, 2009), en ocasiones se justifica el diagnóstico inicial de TEA con la finalidad de acceder a los servicios de atención necesarios (Rice, 2013). Otro tipo de sustitución de diagnóstico puede darse en el caso de las formas más leves de TEA. Los niños que actualmente son diagnosticados con el trastorno de Asperger pueden haber sido diagnosticados previamente con otros diagnósticos psiquiátricos (por ejemplo, trastorno obsesivo-compulsivo, fobia escolar, ansiedad social, etc.) (Fombonne, 2009).

Debemos considerar que las características clínicas de los TEA y la DI se superponen en muchos casos creando una confusión potencial a la hora de su identificación. En un estudio realizado sobre 2.816 niños con DI con y sin TEA (Pedersen y otros, 2016), se observó que los intereses restringidos y las conductas estereotipadas discriminan mejor entre ambos grupos. Sin embargo, es conocido el peso que tiene en el diagnóstico de los TEA la dimensión de la comunicación social. Es importante para la mejora de la asignación del tipo de intervención la adecuada diferenciación, aunque se reconoce que en ocasiones resulta extraordinariamente difícil y se cae en la comorbilidad de síntomas. Según diversos estudios la concurrencia de los diagnósticos de TEA

(C) Ediciones Universidad de Salamanca / CC BY-NC-ND

Siglo Cero, vol. 47 (4), n. ${ }^{\circ} 260,2016$, octubre-diciembre, pp. 7-26

$$
-19-
$$


Figura 6. Evolución del número de subvenciones solicitadas según diferentes tipos de trastornos (datos obtenidos de 24)

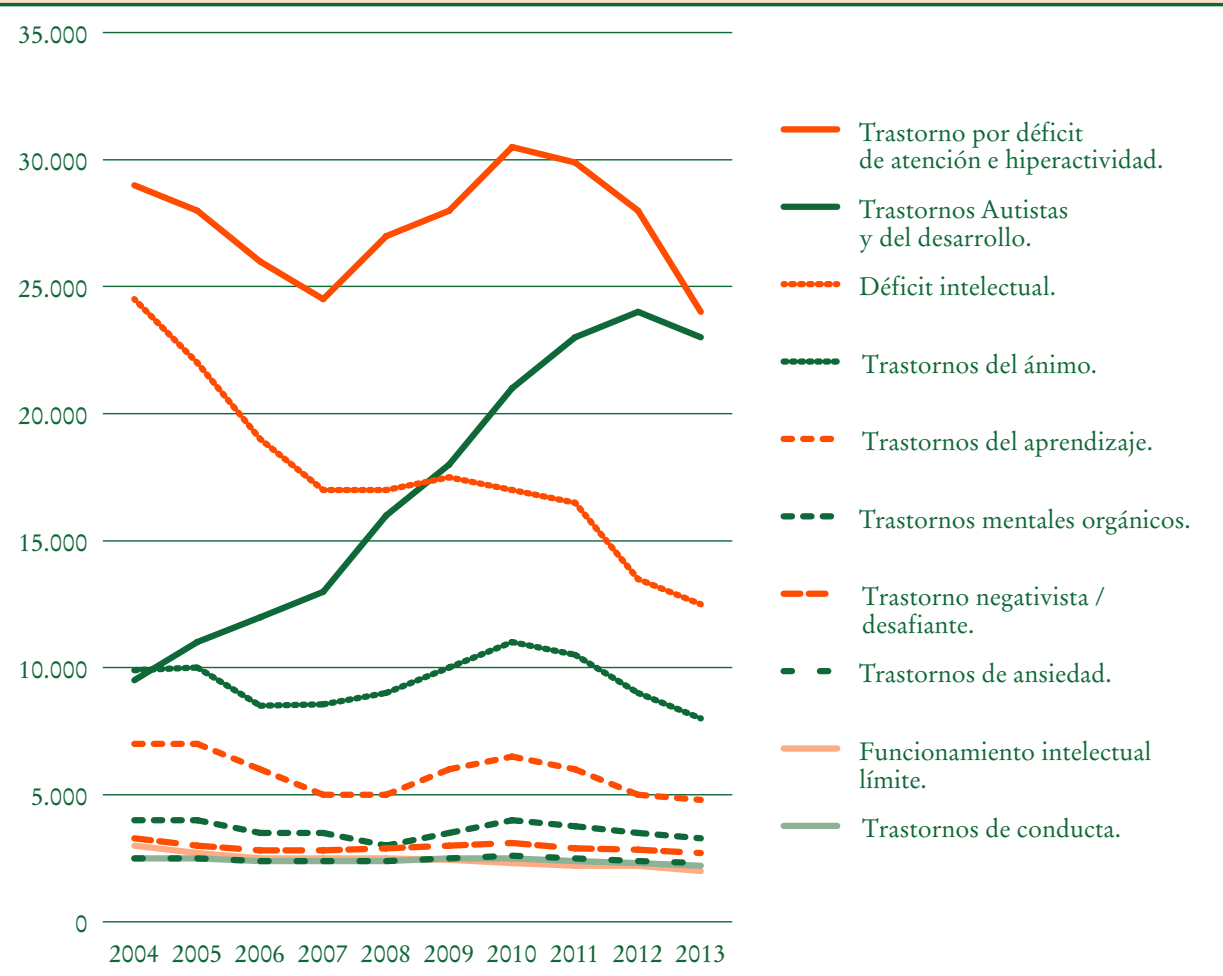

y DI aumenta (Goldberg, 2006) o decrece (Elsabbagh y otros, 2012). Las dificultades en la diferenciación y la falta de recursos para la intervención en niños con DI puede justificar que en casos de diagnóstico concurrente se ponga el acento en el diagnóstico de TEA frente a otros.

\section{Conclusión}

A lo largo del presente artículo se han presentado evidencias del incremento en la tasa de prevalencia de los TEA. Sin embargo, este incremento no es totalmente atribuible a un incremento en la incidencia del trastorno. Aunque la mayoría de los estudios expuestos tienen como origen los datos de USA (tendencia creciente, variabilidad en función de localizaciones geográficas, problemas en la identificación y criterios diagnósticos, etc.) entendemos que, en general, se pueden extrapolar al resto de países occidentales (Elsabbagh y otros, 2012), añadiendo, en el caso de algunos países, razones de índole sociocultural y económica. 
Se han descrito factores como los cambios en los criterios diagnósticos, las prácticas de detección y diagnóstico, las políticas de educación especial, la disponibilidad de servicios y el conocimiento de los TEA (tanto en el público en general como entre los profesionales), pueden ser responsables del aumento de la prevalencia en el tiempo. En la década de los 90 , el aumento en el número de niños diagnosticados produjo simultáneamente en muchos países la expansión significativa de servicios para niños con TEA, provocando un efecto dominó.

Por otra parte, la significación estadística o las limitaciones muestrales de los estudios o posibles sesgos en los procedimientos de selección de casos pueden ser una limitación en la mayoría de las investigaciones; por lo tanto, se pueden detectar variaciones de pequeña magnitud en la incidencia de TEA que deben ser interpretados con precaución.

No obstante, no debe ser eliminada por completo la posibilidad de que un cierto aumento en la incidencia de trastornos del espectro autista también haya contribuido, en parte, a la tendencia al alza en las tasas de prevalencia. Para evaluar si la incidencia se ha incrementado, en los estudios futuros se deben controlar de forma rigurosa los factores metodológicos que, como se ha evidenciado, dan cuenta de una importante proporción de la variabilidad en los datos.

Los futuros programas de vigilancia o aquellos que están en curso actualmente deberán proporcionar datos más significativos para evaluar esta hipótesis. Además, queda por ver cómo los cambios en criterios diagnósticos introducidos en el DSM-5 o futuros cambios de criterio diagnóstico impactarán en las estimaciones de la prevalencia de TEA. Mientras tanto, las cifras de prevalencia disponibles tienen implicaciones directas para las necesidades actuales y futuras de servicios y programas de intervención temprana y el aumento de investigaciones sobre sus posibles causas. Es evidente que las políticas sociales, educativas, sanitarias deben tener en cuenta la situación descrita incidiendo principalmente en el incremento de servicios de atención temprana o psicoeducativa no solo en los niños/as con TEA. De la misma forma, se hace necesario que en el proceso diagnóstico se tenga en cuenta más el perfil de competencias cognitivo para valorar la discriminación entre TEA y Discapacidad Intelectual o la comorbilidad de ambos trastornos. En la actualidad, el peso de los síntomas de TEA evaluados por instrumentos como el ADOS-2 (Lord y otros, 2012) y el ADI-R (Rutter, Le Couteur y Lord, 2009) son quizás sobrevalorados en perjuicio de otros instrumentos que nos permitieran discernir con más precisión entre estos dos trastornos. En USA está tomando cada vez más peso el uso de las escalas Mullen (Mullen, 1995; Bradley-Jonson, 1997; Ozonoff, Gooflin-Jones y Solomon, 2010), escalas no traducidas ni adaptadas a nuestro entorno sociocultural.

\section{Referencias bibliográficas}

Aкshoomoff, N. (2006). Use of the Mullen Scales of Early Learning for the Assessment of Young Children with Autism Spectrum Disorders. Journal of Child Neuropsychology, 12, 4-5. doi:10.1080/09297040500473714. 
Alberdi, J. (1990). Investigación Epidemiológica de niños autistas en el territorio foral de $\mathrm{Na}$ varra. Bilbao: Universidad del País Vasco.

Al-Farsi, Y., Al Farsi, O. y Brooks, D. (2011). Brief Report: Prevalence of Autistic Sprectrum Disorders in the Sultanate of Oman. Journal of Autism and Developmental Disorders, 41, 821-825. doi:10.1007/s10803-010-1094-8.

APA (2013). Diagnostic and Statistical Manual of Mental Disorders 5th ed. Arlington: American Psychiatric Publishing.

BAio, J. (2012). Prevalence of Autism Spectrum Disorders- Autism and Developmental Disabilities Monitoring Network, 14 sites, United States, 2008. Morbidity and Mortality Weekly, 61,1-19. Descargado de http://www.cdc.gov/mmwr/preview/mmwrhtml/ss6103a1.htm.

BAIO, J. (2014). Prevalence of autism spectrum disorder among children aged 8 years: Autism and developmental disabilities monitoring network, 11 sites, United States, 2010. Morbidity and Mortality Weekly Report, 63, 1-21. Descargado de http://www.cdc.gov/mmwr/ preview/mmwrhtml/ss6302a1.htm.

Blaxill, M. (2004). What's going on? The question of time trends in autism. Public Health Reports, 119 (6), 536-551. doi:10.1016/j.phr.2004.09.003.

Blumberg, S., Brankett, M., Kogan, M., Schiever, L., Jones, J. y Lu, M. (2013). Changes in prevalence of parent-reported autism spectrum disorder in school-aged U.S. children: 2007 to 2011-2012. National Health Statistics Reports, 65 (20), 1-12.

Bont, T. y Wu, J. (2015). Mental Disorders and Disabilities Among Low-Income Children. Washington: National Academy Press. Extraído de http://www.ncbi.nlm.nih.gov/books/ NBK332882/.

Bourke, J., De Klerk, N., Smith, T. y Leonard, H. (2016). Population-Based Prevalence of Intellectual Disability and Autism Spectrum Disorders in Western Australia: A comparison with previous estimates. Medicine, 95 (21). doi:10.1097/MD.0000000000003737.

Boyle, C., Boulet, L., Schieve, R., Cohen, S., Blumberg, M., Yeargin-Allsopp, S. y Kogan, M. (2011). Trends in the prevalence of developmental disabilities in U.S. children 19972008. Pediatrics, 127 (6), 1034-1042. doi:10.1542/peds.2010-2989.

Bradley-Jonson, S. (1997). Mullen scales of early learning. Psychology in the Schools, 34 (4), 379-382. doi:10.1002/(SICI)1520-6807(199710)34:4<379::AID-PITS14>3.0.CO;2-E.

Braun, K., Chistensen, D., Doernberg, N., Schieve, L., Rice, C., Wiggins, L., ... YearginAllsopp, M. (2015). Trends in the prevalence of autism spectrum disorder, cerebral palsy, hearing loss, intellectual disability and vision impairmente metropolitan Atlanta 19912010. PLos ONE, 10 (4), e0124120.

Canal, R., García, P., Martín, M., Santos, J., Guisuraga, Z., Herráez, L., ... Posada, M. (2010). Modified Checklist for Autism in Toddlers: Cross-Cultural Adaption and Validation in Spain. Journal of Autism and Developmental Disorders, 41, 1342-1351. doi:10.1007/ s10803-010-1163-z.

Cavagnaro, A. (2007). Autistic spectrum disorders: Changes in the California caseload. An update: june 1987- june 2007. California: California Health and Human Services Agency (Consultado 23-3-2015). Descargado de http://www.dds.ca.gov/Autism/docs/AutismReport_2007.pdf.

Charman, T. (2002). The prevalence of autism spectrum disorders: Recent evidence and future challenges. European Child and Adolescent Psychiatry, 11 (6), 249-256. doi:10.1007/s00787002-0297-8.

Chistensen, D., Baio, J., Braun, K., Bilder, D., Charles, J., Constantino, J., ... YearginAllosop, M. (2016). Prevalence and Characteristics of Autism Spectrum Disorder Among

(C) Ediciones Universidad de Salamanca / CC BY-NC-ND

Siglo Cero, vol. 47 (4), n. ${ }^{\circ}$ 260, 2016, octubre-diciembre, pp. 7-26 
PREVALENCIA DE LOS TRASTORNOS DEL ESPECTRO AUTISTA: REVISIÓN DE DATOS FRANCISCO ALCANTUD MARÍN, YURENA ALONSO ESTEBAN Y SUSANA MATA ITURRALDE

Children Aged 8 Years. Autism and Developmental Disabilities Monitoring Network, 65 (3), 1-23. doi:10.15585/mmwr.ss6503a1.

Christiansz, J., Gray, K., Taffe, J. y Tonge, B. (2016). Autism Spectrum Disorder in the DSM-5: Diagnostic Sensitivity and Specificity in Early childhood. Journal of Autism and Developmental Disorders, 46, 2054-2063. doi:10.1007/s10803-016-2734-4.

Coo, H., Ouellette-Kuntz, H., Lloyd, J., Kasmara, L., Holden, J. y Lewis, M. (2008). Trends in autism prevalence: diagnostic substitutions revisited. Journal of Autism and developmental Disorders, 38, 1036-1046. doi:10.1007/s10803-007-0478-x.

Elsabbagh, M., Divan, G., Kohn, Y., Kim, Y., Kauchali, S., Marcín, C., ... Fombonne, E. (2012). Global prevalence of autism and other pervasive developmental disorders. Autism Research, 5 (3), 160-179. doi:10.1002/aur.239.

Folch, J. y SÁnchez, L. (1998). Estudio epidemiológico del Autismo, las psicosis infantiles y las oligofrenias en la comarca de Osona (Cataluña, España), con un seguimiento longitudinal de veinticinco años (1969-1994). En V Congreso Internacional autismo-Europa (pp. 177-227). Barcelona: Fundación ONCE y Escuela libre editorial. Descargado el 14 de julio de 2016 de http://www.autisme.com/autisme/documentacio/documents/Estudi\%20 epidemiologico $\% 20$ del $\% 20$ autismo $\% 20$ en $\% 20$ la $\% 20$ comarca $\% 20$ de $\% 20$ Osona.pdf.

Fombonne, E. (2003). The prevalence of autism. The Journal of the American Medical Association, 289, 87-89. doi:10.1001/jama.289.1.87.

Fombonne, E. (2007). Epidemiology. En A. Martin y F. Volkmar, Lewis's child and adolescent psychiatry: a comprehensive textbook (pp. 150-171). Philadelphia: Lippincott Williams \& Wilkins.

Fombonne, E. (2009). Epidemiology of perversive developmental disorders. Pediatric Research, 65 (6), 591-598.

Fortea, M., Escandell, M. y Castro, J. (2013). Estimación de la prevalencia de los trastornos del espectro autista en Canarias. Anales de Pediatría, 79 (6), 352-359. doi:http://dx.doi. org/10.1016/j.anpedi.2013.04.022.

Frontera, M. (2005). Estudio epidemiológico de los trastornos generalizados del desarrollo en la población infantil y adolescente de la Comunidad Autónoma de Aragón. Zaragoza: Gobierno de Aragón, Departamento de Educación, Cultura y Deporte. Descargado el 15 de julio de 2016 de http://aetapi.org/download/estudio-epidemiologico-de-los-trastornosgeneralizados-del-desarrollo-en-la-poblacion-infantil-y-adolescente-de-la-comunidadautonoma-de-aragon/?wpdmdl=1155.

Garanto, J. (1994). Epidemiología de la Psicosis y Autismo. En J. Garanto, Epidemiología de las psicosis y del Autismo. Salamanca: Amarú Ediciones.

García-Primo, P., Santos, J., Martín, M., Martínez, M., Lleras, S., Posada de la Paz, M. y Canal, R. (2014). Programa de detección precoz de trastornos generalizados del desarrollo en las áreas de salud de Salamanca y Zamora. Anales de Pediatría, 80 (5), 285-292. doi:10.1016/j.anpedi.2013.06.030.

GillberG, C. (1984). Infantile autism and other childhood psychises in a Swedixh urban region. Epidemiological aspects. Journal of Child Psychological Psychiatry, 25 (1), 35-43. doi:10.1111/j.1469-7610.1984.tb01717.x.

Gillberg, C., Steffenburg, S. y Schaumann, H. (1991). Is autism more common now than ten years ago? British Journal of Psychiatry, 158, 403-409. doi:10.1192/bjp.158.3.403.

Goldberg, M. (2006). Are the Majority of Children With Autism Mentally Retarded? Focus on Autism and Other Developmental Disabilities, 21 (2), 66-83. doi:10.1177/10883576060 210020301.

(C) Ediciones Universidad de Salamanca / CC BY-NC-ND

Siglo Cero, vol. 47 (4), n. ${ }^{\circ} 260,2016$, octubre-diciembre, pp. 7-26 
Hansen, S., Schendel, D. y Parner, E. (2014). Explaining the increase in the prevalence of Autism Spectrum Disorders. The Journal of the American Medical Association Pediatrics, 56-62. doi:10.1001/jamapediatrics.2014.1893.

Hill, A., Zuckerman, K. y Fombonne, E. (2015). Epidemiology of Autism Spectrum Disorders. En M. Robinson-Agramonte, Translational Approaches to Autism Spectrum Disorder (pp. 12-38). New York: Springer. doi:10.1007/978-3-319-16321-5.

IMSERSO (2005). Informe Anual. Madrid: Ministerio de Sanidad, Servicios Sociales e Igualdad. Descargado el 10 de junio de 2016 de https://sede.imserso.gob.es/Sede/portal/homePortal.seam.

INE (2011). Encuesta Nacional de Discapacidad, Autonomía Personal y Situaciones de Dependencia 2008. Madrid: Ministerio de Economía y Competitividad. Descargado el 3 de mayo de 2016 de http://www.ine.es/dyngs/INEbase/es/operacion.htm?c=Estadistica_C\&cid=1 $254736176782 \&$ menu $=$ resultados\&idp $=1254735573175$.

Kim, Y., Leventhal, B., Koh, Y., Fombonne, E., Laska, E., Lim, E., ... Grinker, R. (2011). Prevalence of Autism Spectrum Disorders in a Total Population sample. American Journal of Psychiatry, 168, 904-912. doi: appi.ajp.2011.10101532.

King, M. y Bearman, P. (2009). Diagnostic change and the increased prevalence of autism. International Journal of Epidemiology, 38 (5), 1224-1234.

Klin, A., Klaiman, C. y Jones, W. (2015). Rebajar la edad de diagnóstico del autismo: la neurociencia del desarrollo social afronta un importante problema de salud pública. Revista de Neurología, S3-S11. Descargado de http://www.neurologia.com/pdf/Web/60S01/bnS01S003.pdf.

LAI, M. C. y BARON-COHEN, S. (2015). Identifying the lost generation of adults with autism spectrum conditions. The Lancet Psychiatry, 1013-1027. doi:10.1016/S2215-0366(15)00277-1.

Lord, C., Rutter, M., Di Lavore, P., Risi, S., Gotham, K. y Bishop, S. (2012). Autism diagnostic Observation Schedule, second edition: ADOS-2. Los Angeles: Western Psychological services.

LotTer, V. (1966). Epidemiology of autisc conditions in young children: I. Prevalence. Social Psychiatry, 1, 124-137. doi:10.1007/BF00584048.

LOTTER, V. (1967). Epidemiology conditions in young children: II. Some characteristics of the parents and children. Social Psychiatry, 1, 163-173. doi:10.1007/BF00578950.

Lundström, S., Reichenberg, A., Anckarsäter, H., Lichtemstein, P. y Gillberg, C. (2015). Autism phenotype versus registered diagnosis in Swedish children: prevalence trends over 10 years in general population samples. British Medical Journal, 350, 1-6. doi:http://dx.doi. org/10.1136/bmj.h1961.

Mandell, D., Barry, C., Marcus, S., Xle, M., Shea, K., Mullan, K. y Epstein, A. (2016). Effects of Autism Spectrum Disorder Insurance Mandates on the Treated Prevalence of Autism Spectrum Disorder. Journal of American Medical Association Pediatrics, E1-E7. doi:10.1001/jamapediatrics.2016.1049.

Matson, J. y Kozlowski, A. (2011). The increasing prevalence of autism spectrum disorders. Research in Autism Spectrum Disorders, 5, 418-425. doi:10.1016/j.rasd.2010.06.004.

Montiel-Nava, C. y PeÑa, J. (2008). Epidemiological findings of perversive developmental disorders in Venezuelan study. Autism, 12 (2), 191-202. doi:10.1177/1362361307086663.

Mullen, M. (1995). Mullen Scales of Early Learnng: AGS Edition. Circles Pines, Minnesota: American Guidance Service.

Orte, L., Martínez, M., López, C., Suances, M., Lonzano, F., Belmonte, F., ... Palma, C. (1995). Estudio epidemiológico de trastornos profundos de desarrollo en la Comunidad Autónoma de Murcia. En AETAPI (Ed.), Actas VIII Congreso Nacional AETAPI: La respuesta

(C) Ediciones Universidad de Salamanca / CC BY-NC-ND

Siglo Cero, vol. 47 (4), n. ${ }^{\circ}$ 260, 2016, octubre-diciembre, pp. 7-26 
educativa (pp. 67-85). Murcia: AETAPI. Descargado el 12 de julio de 2016 de http://aetapi. org/download/estudio-epidemiologico-de-trastornos-profundos-del-desarrollo-en-lacomunidad-autonoma-de-murcia/?wpdmdl=1362.

Ouhtit, A., Al-Farsi, Y., Al-Sharbati, M., Waly, M., Gupta, I., Al-Farsi, O., ... Al-Adawi, S. (2015). Underlying Factors Behind the low prevalence of Autism Spectrum Disorders in Oman: Sociocultural perspective. Sultan Qaboos University Medcal Journal, 15 (2), 213 217. doi:PMC4450784.

Ozonoff, S., Gooflin-Jones, B. y Solomon, M. (2010). Evidence-Based Assessment of Autism Spectrum Disroders in Children and Asolescents. Journal of Clinical Child E Adolescent Psychology, 34 (3), 523-540. doi:10.1207/s15374424jccp3403_8.

Pedersen, A., Pettygrove, S., Lu, Z., Andrews, J., Meaney, F., Kurzius-Spencer, M., ... Cunniff, C. (2016, agosto). DSM Criteria that Best Differentiate Intellectual Disability. Child Psychiatry and Human Development, 24. doi:10.1007/s10578-016-0681-0.

Posada de la Paz, M., Ferrari Arroyo, M., Touriño, E. y Boada, L. (2005). Investigación epidemiológica en el autismo: una visión integradora. Revista de Neurologia, 40 (1), S191-S198. Descargado el 14 de julio de 2016 de http://www.neurologia.com/pdf/ Web/40S1/sS1S191.pdf.

Rice, C. (2013). Time trends in diagnosis. En F. Volkmar, Encyclopedia of autism spectrum disorders (pp. 3120-3125). New York: Springer. doi:10.1007/978-1-4419-1698-3_14.

Rutter, M., Le Couteur, A. y Lord, C. (2009). Autism Diagnostic Interview Revised. Los Angeles: Autism Genetic Resource Exchange.

Sadami, S., Mahmoodizadeh, A. y McConkey, R. (2012). A national study of the prevalence of autism among five-year-old children in Iran. Autism, 16 (1), 5-14. doi:10.1177/1362361311407091.

Sadami, S. y McConkey, R. (2011). Autism in Developing Countries: Lessons from Iran. Autism Research and Treatment, 1-11. doi:10.1155/2011/145359.

Saldaña, D., Álvarez, R., Moreno, M., López, A., Lobatón, S. y Rojano, M. (2013). Vida Adulta y trastornos del espectro autista: Calidad de vida y empleo en Andalucía. Sevilla: Federación Autismo Andalucía. Descargado de http://sid.usal.es/22145/8-1.

Sánchez-Valle, E., Posada, M., Villaverde-Hueso, A., Touriño, E., Ferrari-Arroyo, M., BoAda, L., ... Fuentes-Biggi, J. (2008). Estimating the Burden of Disease for Autism Spectrum Disorders in Spain in 2003. Journal of Autism and Developmental Disorders, 38, 288296. doi:10.1007/s10803-007-0393-1.

ShatTUCK, P. (2006). The contribution of diagnostic substitution to the growing administrative prevalence of autism in US special education. Pediatrics, 117 (4), 1028-1037.

Taylor, B., Jick, H. y MacLaughlin, D. (2013). Prevalence and incidence rates of autism in the UK: time trend from 2004-2010 in children aged 8 years. British Medical Journal Open, 3, 1-6. doi:10.1136/bmjopen-2013-003219.

Trujillo, M., SÁnchez, M. y Gutiérrez, M. (2007). Estudio de la prevalencia de los TGD a través de una unidad de salud mental infantil: Un estudio preliminar. Interpsiquis. Psiquiatria.com. Descargado el 15 de julio de 2016 de http://www.psiquiatria.com/bibliopsiquis/ assetstore/16/23/58/162358714569359869181482553499288365240.

WING, L. (1993). The definition and Prevalence of Autism: A Review. European and Adolescent Psychiatry, 2, 61-74. doi:10.1007/BF02098832.

WING, L. y Gould, J. (1979). Severe impairments of Social interation and associated abnormalities in children: Epidemiology and classification. Journal of Autism and developmental Disorders, 9, 11-29.

(C) Ediciones Universidad de Salamanca / CC BY-NC-ND

Siglo Cero, vol. 47 (4), n. ${ }^{\circ}$ 260, 2016, octubre-diciembre, pp. 7-26 
PREVALENCIA DE LOS TRASTORNOS DEL ESPECTRO AUTISTA: REVISIÓN DE DATOS FRANCISCO ALCANTUD MARÍN, YURENA ALONSO ESTEBAN Y SUSANA MATA ITURRALDE

Wing, L. y PotTer, D. (2002). The epidemiology of autism spectrum disorders: Is the prevalence rising? Mental Retardation and developmental Disabilities Research Reviews, 8 (3), 151-161. doi:10.1002/mrdd.10029.

Wong, V. y Hui, S. (2008). Epidemiological Study of Autism Spectrum Disorder in China. Journal of Child Neurology, 23 (1), 67-72. doi:10.1177/0883073807308702.

(C) Ediciones Universidad de Salamanca / CC BY-NC-ND

Siglo Cero, vol. 47 (4), n. ${ }^{\circ}$ 260, 2016, octubre-diciembre, pp. 7-26 